\title{
A Virtual Instructor System for Supervising the Physical Exercises of Patients with Arthritis
}

\author{
Pranav Badhe ${ }^{1}$, Sachin Gawate ${ }^{2}$ \\ ${ }^{1}$ M.E. Student, Embedded System and Computing, G.H Raisoni College of Engineering Nagpur, Maharashtra, India \\ ${ }^{2}$ Assistant Professor, Department of Computer Science and Engineering, G .H Raisoni College of Engineering Nagpur, Maharashtra, India

\begin{abstract}
Arthritis is joint related disease which is largely due to the nodule that emerges on the joints of person and it also decrepitude the joints of body. This disease can foster the heart diseases and can also provoke the sabotage of vital organs. Physical activities and also the physiotherapy can spark off the pain which is useful to medicate the disease. Person do not get the avail from the prescript practice and there are abstruse instruction also. Patients does not follow the exercises which are recommended to them decently. The patients who pay a visit to Medical Centre might not earn a personal dedicated trainer to supervise the training and this might affect the restoration from the disease. This paper ventures a technique that is useful in bringing a virtual instructor to observe the recommended exercises and also to teach the routine exercises.
\end{abstract}

Keywords: Microsoft Kinect, Depth image, motion tracking, Skelton tracking, Arthritis

\section{Introduction}

Arthritis can influence the human body joints and it can also affect the motion of these joints. Arthritis also is the prime reason for the pain of the joints and also the nodule of the joints. The patients with acute level of arthritis can have the resolute pain and they also find it very difficult to do the daily activities and this affects their daily life. Arthritis might also be the main reason behind the rigid changes in the joints. The defacement caused by the arthritis can only be seen by the X-ray. Arthritis also affects the skin, lungs and other organs of the body. It can also cause the eruption in the lungs and it also arises the risk of the diseases related to the heart. Figure 1 shows the joints of body with Arthritis.

The foremost aim of the physical exercises is to increase the motion of the body of the person suffering from the arthritis. The improvement of the motion of the joints and the strengthening of the muscle force can be uplift by the recommended therapy made up of the appropriate exercises. The physical therapy is the pivotal reason for the enhancement of the daily life of the person who is suffering from the arthritis. Patients with arthritis ought to do the physical exercises frequently to minimize the destructive effects caused by the disease. The person who follows the daily recommended exercises exhibits a great improvement in the motion of the joints and also in the strength of the muscles and also improves the blood pressure condition.

For the real time tracking of the motion the convenient systems are the 3D motion tracking systems. The 3D motion tracking are far better in comparison to the $2 \mathrm{D}$ motion tracking in terms of the precision and the effectiveness. But these systems aren it used in daily basis as it is very tangled in few systems and also it requires an excessive cost and it may also not serve the user friendliness. This factors affects usage of these systems in daily life. Because of these factors most of the clinics prefers the traditional manual methods.

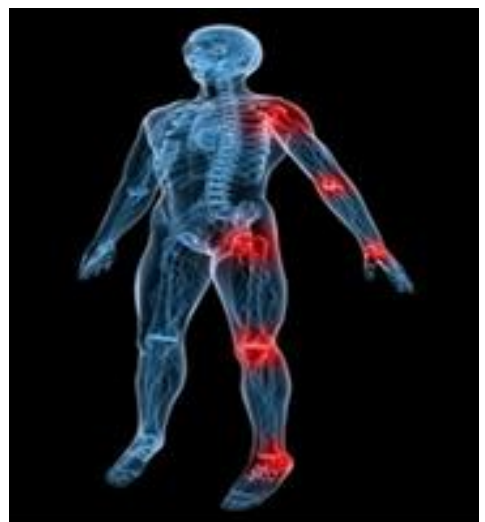

Figure 1: Joints of human body suffering from Arthritis

Due to the excessive cost, it is not possible to be used by the majority of the people. People may not get the personal dedicated trainer for the $\mathrm{m}$ while they visit the physiotherapy clinics. If the people do not follow the recommended sets of exercises accurately and regularly then this will boost the harmfulness of the disease and will cause the adverse effects to the patient's health. There are chances that the patients do not follow the ideal exercises. Above factors are the prime reason for implementing the cost effective systems that can supervise the exercise routines of the patients.

In this paper, a technique is presented which amplify the recovery of the patients by providing a robust method to supervise and tutor the recommended exercise routines to the arthritis patients. Kinect sensor is used for the motion tracking in real time. The human motion tracking is done by using the skeleton tracking method. The system is integrated to captivate the patient to perform the recommended routine exercises. A virtual instructor is provided by this system to tutor the exercise activities to the patient who is suffering from the arthritis. 


\section{International Journal of Science and Research (IJSR) \\ ISSN (Online): 2319-7064}

Index Copernicus Value (2013): 6.14 | Impact Factor (2015): 6.391

\section{Literature Survey}

In 2013 Jing Chen, Baoliang Wang, Zeyu Chen [1], tendered a technique to track the gesture using the Kinect. The authors makes the different shape using both the hand. They track the shapes made by the hands of the person in front of the Kinect. Then they finds out the shape made by the person in front of the Kinect.

In 2014 Ondrej Kainz, František Jakab [3], proposed a way for tracking the hand and the gesture with the help of the surface electromyography. This method is capable of boosting the rate of the identification of the gesture. The depth image data is used by the system and the system electric agitation also used the triggered from the muscle of forearm. This methods is also capable of tracking the hand even when the obstacle arrives to cover it.

In 2012, Dongheui Lee, Jurgen Sturm, Licong Zhang, Daniel Cremers, [4] proposed a technique using more than one Kinect for motion tracking. This technique competent of tracing the pose of the human precisely. The real time 22DOF human pose using GPGPU support is used for the tracing of the pose of the human.

In 2015 Aeri Rachmad, Muhammad Fuad [5], proposed a method which is capable of giving the data of a particular gesture with the help of the peculiar tools like rods and flags. Kinect sensor is used to secure the data related to the skeleton image and elaborates the algorithm for the tracing of the gesture.

\section{Framework}

The key motive for the project is to evolve a system which is capable of teaching the recommended exercises and also supervise it precisely in real time. The recommended exercises will be mentored by this system in a great way to the patients. The matching of the ideal exercise pattern with the exercises performing in real time will be done by this system accurately.

Kinect sensor is used in this project for the real time motion tracking. The Kinect is capable of performing the skeleton tracking and depth sensor techniques. Kinect techniques plays a crucial role in tacking the motion in real time precisely and effectively.

Kinect is evolved for the Xbox and Windows PCes by Microsoft [3]. The characteristics of the Kinect are the depth image capability along with the multi array microphone and RGB camera [3] [14]. Kinect has the output frame rate from $9 \mathrm{~Hz}$ to $30 \mathrm{~Hz}$ which is established on the resolution. The Kinect sensor range is from 1.2 to $3.5 \mathrm{ft}$. The Microsoft SDK is essential for using the Kinect. Fig shows Microsoft Kinect.

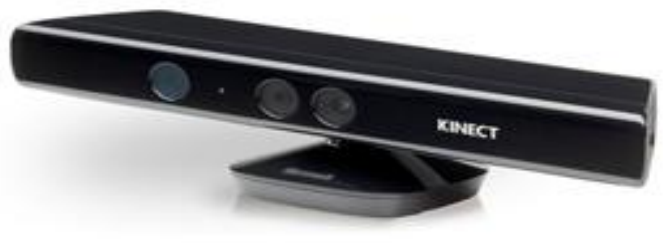

\section{Working}

Fig Microsoft Kinect

The principal purpose of the system is revealed by the block diagram. In the first place the system will look to withdraw the persones image with the help of the Kinect sensor. Then the system will create the database by using the withdrawn image of the person doing the ideal pattern of exercises. After that the system will attain the pattern of the line skeleton with the help of the skeleton tracking method. After that the co-ordinates of the joints will be calculated from the skeleton tracking technique. Then the ideal line skeleton pattern will be forecasted by the system. After that the real time skeleton tracking will be carried out and matched with the database pattern. Then the unification of both the hardware and software will carried out. The programming of the system is done by using the MATLAB software.

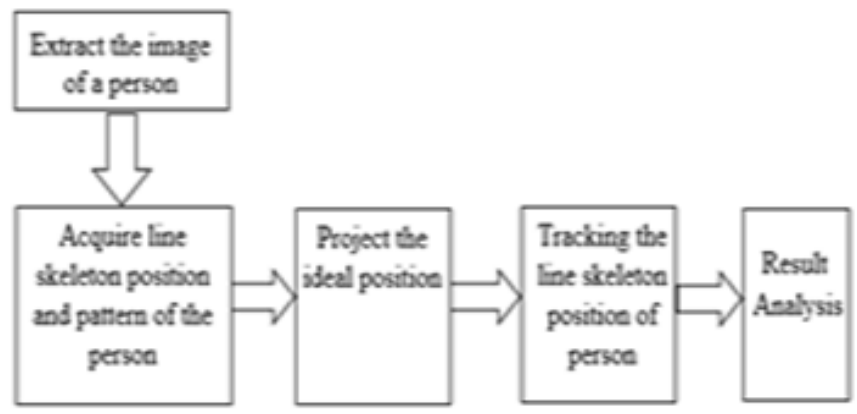

Figure 2: Block diagram

The Kinect camera is activated by a switch near the person who want to activate the system. When the switch is pressed it will send the initializing signal to the system and then the system will be turned on. The main reason for the use of the switch is to help the person to initialize the system who is standing at a distance to come in the range of the sensor and the person will not have to go near the system to turn it on. And the person and perform the exercises from the recommended place so that the complete body of the person will be tracked by the Kinect sensor. 


\section{International Journal of Science and Research (IJSR)}

ISSN (Online): 2319-7064

Index Copernicus Value (2013): 6.14 | Impact Factor (2015): 6.391

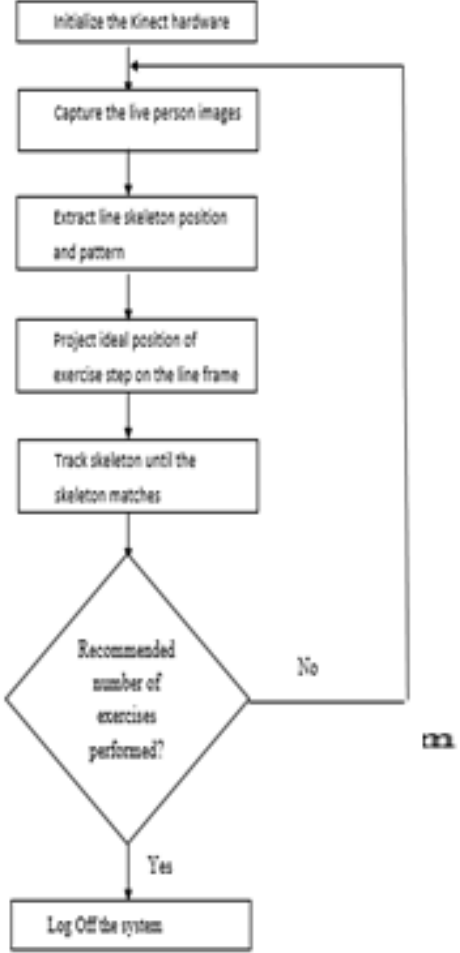

Figure 3: Working flow chart

Then after the system is initialized then it will begin to create the database by tracking the recommended exercise for the patient by tracking the live skeleton pattern of the person performing the exercises ideally. After that the Kinect will create the database of these exercises by saving the coordinates of the joints by using the ideal skeleton pattern. The Kinect contains of an infrared emitter which projects the unique pattern and the reflection of these pattern is captured by the infrared sensors [10].This helps in acquiring the joint co-ordinates from the depth image created by the sensor. These depth co-ordinates are afterwards transformed into the skeleton co-ordinates. The joints of human body is tracked by the skeleton tracking technique. It is proficient of tracking the 20 joints. It can track the line skeleton of the person not only in standing position but also in sitting position. Kinect is proficient of tracking the 10 joints in the sitting position. Skeleton patterns of the six persons can be achieved simultaneously. Fig 4 shows the skeleton tracking in real time.

When the system gets the ideal skeleton pattern and position of the recommended exercise, it will create a required threshold by projecting the ideal pattern and real time skeleton tracking of person performing the same exercise in real time.

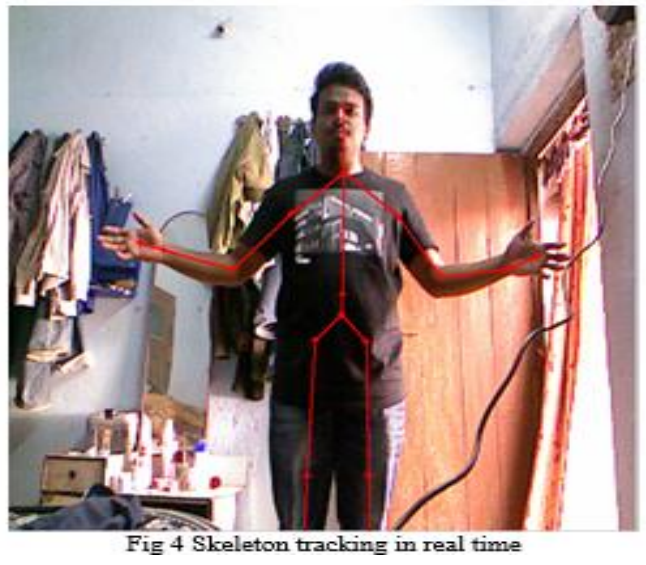

The threshold will be created by comparing the ideal exercise pattern from the database with the real time skeleton pattern of the person performing the same exercise pattern. The difference of the coordinates of the joints of the human body of the skeleton pattern and the live skeleton pattern of the person performing the similar exercise will lead to distinguish the required threshold. . Fig 5 shows skeleton matching of ideal and real time patterns.

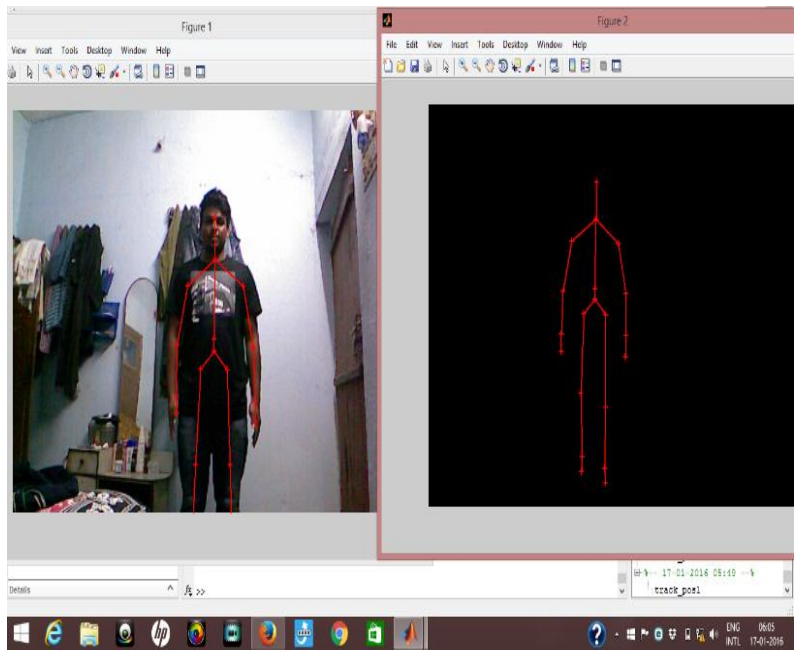

Figure 5: skeleton matching of ideal and real time patterns.

With the help of the database the system will create a routine of exercises which are recommended to the patients. The person can run the particular program of exercise recommended to him by the consulting doctor. Like if a person has a knee arthritis the he can select the shoulder arthritis exercises from the system and the elbow arthritis patient can select the exercises related to the elbow. After that the patient have to go the recommended position from the camera so that the whole body of that particular patients can be captured by the camera and the patients can comes in the range of the camera. Then the patient can initialize the system by pressing a switch which provided near the recommended position from the camera. After pressing the switch the system will initialized and the Kinect will start to work. Later the real time skeleton tracking is carried out and the tracked skeleton is shown in red color and at the same time the virtual instructor in the form of a green color skeleton showing the recommended exercise also gets forecasted. The live skeleton to guide is in green color which helps the patient to perform the prescribed exercise in great 


\section{International Journal of Science and Research (IJSR) \\ ISSN (Online): 2319-7064 \\ Index Copernicus Value (2013): 6.14 | Impact Factor (2015): 6.391}

way. In this way the person can track his skeleton pattern with the projected skeleton pattern can match the projected pattern and perform the set of recommended exercises. After the ideal pattern is matched with the real time pattern then the virtual instructor projects the next exercise pattern and so on until the recommended sets of exercises are not performed. This way the virtual instructor is capable of matching the prescribed exercises as well as supervise the patient performing the recommended exercises in real time.

\section{Result}

The recommended number of reputations of the exercises will also be supervised by the virtual instructor. Hence the virtual instructor is capable of entirely supervising the prescribed exercise routine. This will also motivate the patients to do the exercise as they can enjoy doing the exercises with the experience of the virtual reality and also can see their progress and this will lead in curing the disease in a great way. Fig $3 \& 4$ shows the prescribed elbow exercises

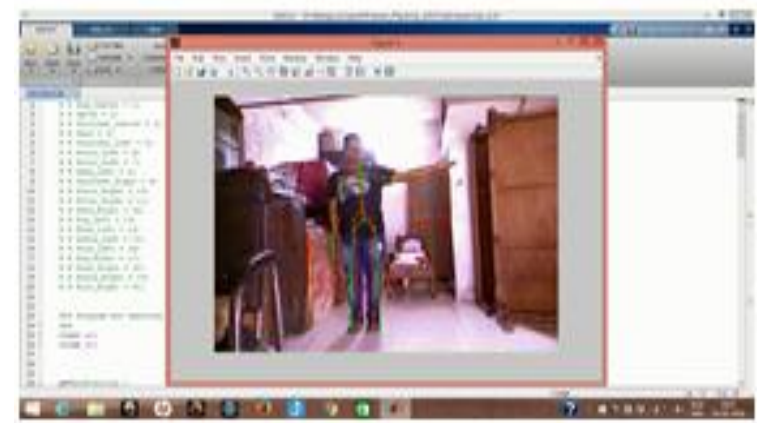

Fig 6: First prescribed elbow exercise

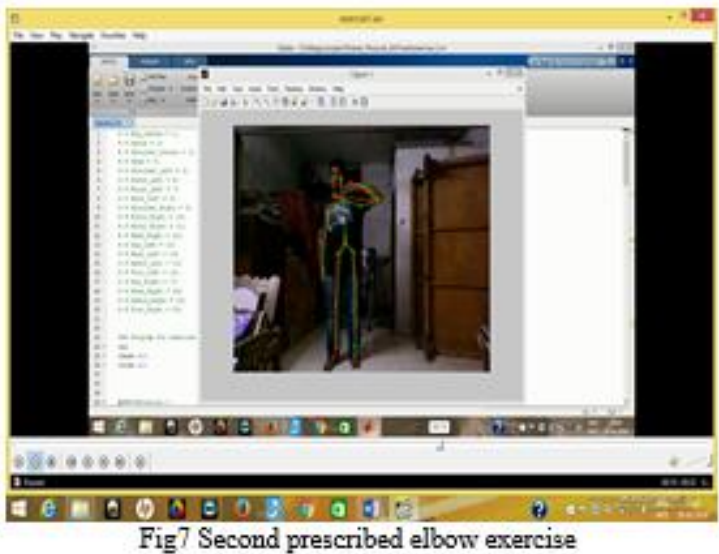

\section{Conclusion}

In this paper, a way is tendered that is capable of supervising and tutoring the exercises to the patient suffering from arthritis with the help of the virtual instructor. The recovery of the patients suffering from arthritis is not only get boost up but also is enchanting the patient to follow the prescribed exercises because of the augmented reality experience. The endorsed exercises are tutored and supervised by this system in real time. It will forecast the first exercise and as soon as the patients manages to complete the first exercise then the system will forecast the new exercise to be performed by the patient. Hence the entire exercise routine can be supervised by this virtual instructor system.

\section{Acknowledgement}

I sincerely thank the HOD of Computer Science Engineering Department, Dr. L. G. Malik for their motivation, inspiration. I would like to thank Assistant Prof. Sachin Gawate for his kind support, guidance and encouragement.

\section{References}

[1] Baoliang Wang, Zeyu Chen, Jing Chen, "Gesture Recognition by Using Kinect Skeleton Tracking System", 2013 Fifth International Conference on Intelligent Human-Machine Systems and Cybernetics 978-0-7695-5011-4/13.

[2] Niranjan Deokule, Geetanjali Kale," Human Action Recognition Using Kinectere, International Journal of Engineering and Computer Science ISSN: 2319-7242 Volume 3 Issue 7 July, 2014 Page No. 7199-720.

[3] Ondrej Kainz, František Jakab," Approach to Hand Tracking and Gesture Recognition Based on DepthSensing Cameras and EMG Monitoring" Acta Informatica Pragensia3(1), 2014, 104-112, DOI: 10.18267/j.aip.38

[4] Licong Zhang, Jurgen Sturm, Daniel Cremers, Dongheui Lee, , ,Real-time Human Motion Tracking using Multiple Depth Cameras", IEEE/RSJ International Conference on Intelligent Robots and Systems, October 7-12, 2012.

[5] Aeri Rachmad, Muhammad Fuad, "Geometry Algorithm on Skeleton Image Based Semaphore Gesture Recognition" Journal of Theoretical and Applied Information Technology 10th November 2015. Vol.81. No.1.

[6] R Campbell, M Evans, M Tucker, B Quilty, P Dieppe, J L Donovan, "Why don't patients do their exercises? Understanding non-compliance with physiotherapy in patients with osteoarthritis of the knee"J EpidemialCommunityHealth2001;55:132-138 doi:10.1136/jech.55.2.132

[7] A. Baillet, E. Payraud, V. A. Niderprim, M. J. Nissen, B. Allenet,P. Franois, L. Grange, P. Casez, R. Juvin, and P. Gaudin, "A dynamic exercise programme to improve patients disability in rheumatoid arthritis:a prospective randomized controlled trial," Rheumatology, vol. 48,no. 4, pp. 410-415, 2009.

[8] M. D. Iversen, A. Finckh, and M. H. Liang, "Exercise prescriptions for the major inflammatory and noninflammatory arthritis," Exercise In Rehabilitation Medicine, pp. 157-179, 2005.

[9] Moiz A. Hussain, G. U. Kharat., ,„,Robust Human Motion Detection and Tracking in Dynamic Background $^{\text {eee }}$ International Journal of Soft Computing and Engineering (IJSCE) ISSN: 2231-2307, Volume-2, Issue-6, January 2013

[10] Chanjira Sinthanayothin, Nonlapas Wongwaen, Wisarut Bholsithi, "Skeleton Tracking using Kinect Sensor \& Displaying in 3D Virtual Scene" International Journal of 
Advancements in Computing Technology(IJACT) Volume4, Number11, June 2012 doi: 10.4156/ijact.vol4.issue 11.23

[11] Ming Zeng; Zhengcun Liu; Qinghao Meng; Zhengbiao Bai;Haiyan Jia, "Motion capture and reconstruction based on depth information using Kinect," Image and Signal Processing (CISP), 2012 5th International Congress on, vol., no.,pp.1381,1385, 16-18 Oct. 2012.

[12] Patsadu, O.; Nukoolkit, C.; Watanapa, B., "Human gesture recognition using Kinect camera," Computer Science and Software Engineering (JCSSE), 2012 International Joint Conference on , vol., no., pp.28,32, May 30 2012-June 12012

[13]Zhang, Zhengyou. "Microsoft kinect sensor and its effect."Multimedia, IEEE 19.2 (2012): 4-10

[14] “msdn.microsoft.com”,https://msdn.microsoft.com/enus/1 ibrary/hh438998.aspx

[15]Lu Xia; Chia-Chih Chen; Aggarwal, J.K., "Human detection using depth information by Kinect," Computer Vision and Pattern Recognition Workshops (CVPRW), 2011 IEEE Computer Society Conference on , vol., no., pp.15,22, 20-25June 2011 